\title{
Interleukin-10 regulates the inflammasome-driven augmentation of inflammatory arthritis and joint destruction
}

\author{
Claire J Greenhill', Gareth W Jones ${ }^{1}$, Mari A Nowell', Zarabeth Newton ${ }^{1}$, Ann K Harvey ${ }^{1}$, Abdul N Moideen ${ }^{1}$, \\ Fraser L Collins', Anja C Bloom', Rebecca C Coll², Avril AB Robertson ${ }^{3}$, Matthew A Cooper ${ }^{3}$, Marcela Rosas', \\ Philip R Taylor ${ }^{1}$, Luke A O'Neill ${ }^{2}$, Ian R Humphreys ${ }^{1}$, Anwen S Williams ${ }^{1}$ and Simon A Jones ${ }^{1 *}$
}

\begin{abstract}
Introduction: Activation of the inflammasome has been implicated in the pathology of various autoinflammatory and autoimmune diseases. While the NLRP3 inflammasome has been linked to arthritis progression, little is known about its synovial regulation or contribution to joint histopathology. Regulators of inflammation activation, such as interleukin (IL)-10, may have the potential to limit the inflammasome-driven arthritic disease course and associated structural damage. Hence, we used IL-10-deficient (IL-10KO) mice to assess NLRP3 inflammasome-driven arthritic pathology.
\end{abstract}

Methods: Antigen-induced arthritis (AIA) was established in IL-10KO mice and wild-type controls. Using histological and radiographic approaches together with quantitative real-time PCR of synovial mRNA studies, we explored the regulation of inflammasome components. These were combined with selective blocking agents and ex vivo investigative studies in osteoclast differentiation assays.

Results: In AIA, IL-10KO mice display severe disease with increased histological and radiographic joint scores. Here, focal bone erosions were associated with increased tartrate-resistant acid phosphatase (TRAP)-positive cells and a localized expression of IL-1 $\beta$. When compared to controls, IL-10KO synovium showed increased expression of II16, II33 and NLRP3 inflammasome components. Synovial NIrp3 and Casp1 expression further correlated with Acp5 (encoding TRAP), while neutralization of IL-10 receptor signaling in control mice caused increased expression of NIrp3 and Casp1. In ex vivo osteoclast differentiation assays, addition of exogenous IL-10 or selective blockade of the NLRP3 inflammasome inhibited osteoclastogenesis.

Conclusions: These data provide a link between IL-10, synovial regulation of the NLRP3 inflammasome and the degree of bone erosions observed in inflammatory arthritis.

\section{Introduction}

As new biologics enter the clinical arena and advances in synovial histopathology identify divergent mechanisms of arthritis progression, it is essential to understand how the cytokine network governs the pattern of synovial inflammation $[1,2]$. Innate sensing mechanisms involving pattern recognition receptors are increasingly implicated in autoimmunity and promote cytokine responses associated with

\footnotetext{
* Correspondence: jonessa@cf.ac.uk

${ }^{1}$ Cardiff Institute of Infection and Immunity, The School of Medicine, Cardiff University, Heath Park Campus, Cardiff CF14 4XN, Wales, UK

Full list of author information is available at the end of the article
}

rheumatoid arthritis. Although these pathways represent promising therapeutic targets, further investigation is required to understand the expression and functional contribution of pattern recognition receptors in autoimmunity.

Pattern recognition receptors were initially characterized as sensors of microbial products of bacterial, fungal or viral infection. These include the Toll-like receptors (TLR); nucleotide-binding domain and leucine-rich repeat containing receptors (NLR), Rig-I-like receptors (RLR) and C-type lectins $[3,4]$. Activation of these receptors promotes the inflammatory regulation of various interleukins, tumour necrosis family members and type-1 interferons 
[3]. They therefore represent innate sensing mechanisms, which shape the adaptive immune response to chronic disease, allergy, cancer and infection. As a consequence, various processes have evolved to protect against the prolonged activation of these receptors. For example, interleukin (IL)-10 limits the duration and intensity of their signaling in myeloid cells [5,6]. Here, IL-10 inhibits pattern recognition receptor signaling through mechanisms, which include downregulation of MyD88 expression [7], and the ubiquitination and subsequent degradation of MyD88-dependent signaling molecules such as TRAF6 [8]. In experimental models of inflammatory arthritis, IL10 is protective and mice deficient in IL-10 show exacerbated joint inflammation $[9,10]$. These data are consistent with the characterization of IL-10 as a cytokine synthesis inhibitory factor, which acts as an immunomodulatory cytokine affecting both innate and cellular immunity $[11,12]$. For example, IL-10 inhibits nuclear factor kappa B (NF- $\mathrm{kB}$ ) signaling in response to TLR agonists to block expression of certain proinflammatory mediators associated with arthritis progression. Interleukin-10 is abundantly expressed in synovial fluids of RA patients and has been linked with the control of bone resorption through inhibition of osteoclastogenesis in vitro [13-15].

While IL-10 is renowned for its ability to inhibit TLR signaling, its impact on innate sensing receptors, including the NLR family, is less documented. Here, caspase 1 activity is essential for the processing of cytokine precursors (for example, pro-IL-1 $\beta$, pro-IL-18 and potentially pro-IL33) into functionally active mature forms [16]. Activation of caspase 1 by the NLR family member NLRP3, acting in association with its adaptor protein ASC, leads to the secretion of IL-1 $\beta$ and IL-18 [16]. Each of these cytokines affect arthritis progression $[17,18]$, which suggests that innate sensing complexes (termed the inflammasome) must be regulated during the course of disease. Various particulate and crystalline agonists activate the NLRP3 inflammasome. For example, monosodium urate crystals and calcium pyrophosphate dihydrate crystals trigger arthritis symptoms in inflammatory forms of gout and pseudogout [19-21], while basic calcium phosphate (hydroxyapatite) crystals are seen in $70 \%$ of osteoarthritis (OA) cases $[22,23]$. A role for the NLRP3 inflammasome in arthritic disease is illustrated by several in vitro studies, which show that crystals from the joints of OA patients and basic calcium phosphate crystals induce IL-1 $\beta$ production by macrophages $[24,25]$. Also, a recent report shows that there is modulation of the NLRP3 inflammasome in peripheral blood mononuclear cells in RA patients and that single nucleotide polymorphisms (SNPs) in NLRP3 are associated with disease severity [26]. However, little is known about the regulation and activation of inflammasome components in inflammatory arthritis. We now show that the exacerbated joint pathology seen in IL-
$10 \mathrm{KO}$ mice during antigen-induced arthritis (AIA) is associated with increased synovial expression of NLRP3 inflammasome components and a localized expression of IL-1 $\beta$ at sites of focal bone erosions. Our data supports a role for IL-10 as a negative regulator of the inflammasome and highlights a role for the inflammasome in osteoclastogenesis during inflammatory arthritis.

\section{Materials and methods}

\section{Mouse strains}

Inbred C57BL/6 mouse strains from The Jackson Laboratory (Bar Harbor, ME, USA) were bred and maintained in-house under high barrier and pathogen-free conditions. All animal studies were performed in the United Kingdom. Experiments were performed on eightto twelve-week-old male mice in accordance with UK Home Office Project License PPL-30/2361 and 30/2928. The ethical approval of these licenses covers all aspects of the study and all experiments conducted.

\section{Induction of murine AIA}

Mice were immunized (subcutaneous (s.c.)) with an emulsion containing $1 \mathrm{mg} / \mathrm{ml}$ methylated bovine serum albumin (mBSA) in phosphate-buffered saline (PBS) and Freud's complete adjuvant (CFA) (Sigma-Aldrich, St Louis, $\mathrm{MO}, \mathrm{USA}$ ). Concurrently, mice were injected (intraperitoneal (i.p.)) with $200 \mathrm{ng}$ of heat-inactivated Bordetella pertussis toxin adjuvant (Sigma-Aldrich, Poole, UK). The immune response was boosted one week later with a second injection (s.c.) of mBSA emulsified in CFA. Arthritis was induced two weeks later with an intra-articular (i.a.) injection of $10 \mu \mathrm{l}$ of $\mathrm{mBSA}(10 \mathrm{mg} / \mathrm{ml})$ into the right knee joint. Arthritis progression was monitored using a micrometer to measure changes in knee joint swelling.

\section{Radiology}

A Kodak in vivo Imaging System FX was used to take radiographs of the mouse knee joints. Both arthritic (right) and non-arthritic joints (left) were compared. Radiographic scores were independently assigned by an orthopedic registrar and based on visible bone erosions (0; normal, 1; mild, 2; moderate, 3; severe).

\section{Histology}

Joints were fixed in neutral-buffered formalin saline, decalcified with formic acid at $4^{\circ} \mathrm{C}$ and embedded in paraffin. Midsagittal sections $(8 \mu \mathrm{m})$ were stained with haematoxylin, safranin-O and Fast Green. Two independent observers scored histology sections for subsynovial inflammation $(0=$ normal, to $5=$ ablation of adipose tissue due to leukocyte infiltrate), synovial exudate $(0=$ normal, to $3=$ substantial number of cells with large fibrin deposits), synovial hyperplasia $(0=$ normal 1 to 3 cells thick, to $3=$ over three layers thick with overgrowth onto joint surfaces with evidence of 
cartilage/bone erosion), cartilage/bone erosion $(0=$ normal, $3=$ destruction of a significant part of the bone). Cartilage integrity was determined in histological sections using a Mankin scoring system. Two independent observers evaluated cartilage irregularity and cleft formation $(0=$ normal, to 6 = complete disorganization of glycoproteins with clefts into the cartilage), cellularity $(0=$ normal, to $3=$ hypocellularity), proteoglycan depletion $(0=$ normal, to $4=$ complete proteoglycan degradation with no dye apparent) and tidemark integrity $(0=$ intact, to $1=$ tidemark crossed by blood vessels). The total sum of these scores resulted in a maximum score of 14. For detection of tartrate-resistant acid phosphatase (TRAP) activity, slides were rehydrated after decalcification, incubated with TRAP staining solution (0.2 M acetate buffer, $50 \mathrm{mM}$ sodium tartrate, $0.5 \mathrm{mg} / \mathrm{ml}$ naphthol AS-MX phosphate, $1.1 \mathrm{mg} / \mathrm{ml}$ Fast Red Violet LB salt) and counterstained with haematoxylin.

\section{Immunohistochemistry}

Antigen retrieval was performed on paraffin-embedded sections using either trypsin $(0.1 \%)$ for 30 mins at $37^{\circ} \mathrm{C}$, or $10 \mathrm{mM}$ citrate buffer $(\mathrm{pH} \mathrm{6})$ for 40 mins at $95^{\circ} \mathrm{C}$. Endogenous peroxidase and biotin activity was blocked using $3 \% \mathrm{H}_{2} \mathrm{O}_{2}$ and an avidin/biotin blocking kit (Vector Laboratories, Burlingame, CA, USA) respectively. Sections were incubated in $10 \%$ (v:v) rabbit serum for 1 hour before staining with rat anti-mouse F4/80 (1:50 dilution, Santa Cruz Technology, Santa Cruz, CA, USA). Antibody binding to sections was detected with rabbit anti-rat biotin-conjugated secondary antibody and streptavidinhorseradish peroxidase (HRP) complex (Vector Laboratories). Diaminobenzidine substrate (Dako, Glostrup, Denmark) was used to develop sections and haematoxylin was used as a counterstain.

\section{Image analysis}

Immunohistochemistry was viewed with a Leica DMLB light microscope (Milton Keynes, UK). Analysis across five random fields of view was performed using the Leica digital image capture program. Values are expressed as a percentage of total immunoperoxidase staining.

\section{Osteoclast cell culture and TRAP stain}

Bone marrow cells from femurs of WT and IL-10KO mice were re-suspended in alpha minimum essential medium ( $\alpha$ MEM) supplemented with 10\% (v:v) foetal calf serum (FCS) and seeded at a density of $6.4 \times 10^{6}$ cells $/ \mathrm{ml}$ in 24-well plates. Following adhesion culture media was supplemented with macrophage colony-stimulating factor (MCSF) and receptor activator of NF-k $\beta$ ligand (RANKL). IL-10 and inflammasome inhibitors were added as indicated in figure legends. TRAP-positive cells were detected after seven days and total RNA isolated from subsequent analysis.

\section{Quantitative real-time PCR (qPCR)}

Synovial membranes were dissected from the underpinning cartilage of knee joints [27]. Total RNA was extracted from samples using TRI Reagent (Sigma-Aldrich) and cDNA derived from $1 \mu \mathrm{g}$ of total RNA using a reverse transcription kit (Primer Design, Southampton, UK) [27]. Gene expression analyses were performed on triplicate samples with SYBR Green (Invitrogen, Thermo Fisher Scientific, Carlsbad, CA, USA) using an ABI Prism 7900HT instrument (Applied Biosystems, Thermo Fisher Scientific, Carlsbad, CA, USA). Details of oligonucleotide primer sequences are presented in Additional file 1. Data analysis was performed using the Sequence Detection System Version 2.3 software (Applied Biosystems).

\section{Enzyme-linked immunosorbent assay (ELISA)}

Serum dickkopf-1 (DKK1) was quantified using a commercial enzyme-linked immunosorbent assay (ELISA) (R\&D Systems Inc, Minneapolis, MN, USA) as per the manufacturer's instructions.

\section{Statistical analysis}

Data was evaluated using the non-parametric MannWhitney $U$ test and an unpaired Student $t$ test. In all cases, $P<0.05$ was considered significant.

\section{Results}

IL-10KO mice display increased disease severity in experimental arthritis

In line with an anti-inflammatory role for IL-10 in experimental arthritis, AIA in IL-10KO mice caused increased joint swelling and an overall exacerbation of joint pathology as compared to wild-type (WT) controls (Figure 1A and B). Evaluation of synovial inflammation based on synovitis, cellular infiltrate, exudate and joint damage showed that histopathology was more prolonged in IL-10KO mice (Figure 1C). Thus, IL-10 acts to prevent long-term subclinical disease and the promotion of self-limiting synovitis.

\section{IL-10-deficiency is associated with loss of cartilage and increased bone erosion}

To determine the impact of joint inflammation on degenerative processes within the inflamed synovium, cartilage damage was based on a Mankin's score and quantified as a percentage loss of safranin-O staining (Figure 2A). When compared to WT mice, cartilage depletion was more pronounced in IL-10KO mice at day 28 and 35 post arthritis induction (Figure 2A). Histology sections from IL-10KO mice also showed evidence of increased bone damage and sites of focal bone erosions. While no significant difference in the quantification of synovial F4/80 staining was observed in synovial tissue for WT and IL$10 \mathrm{KO}$ mice, an increased localization of F4/80 staining 

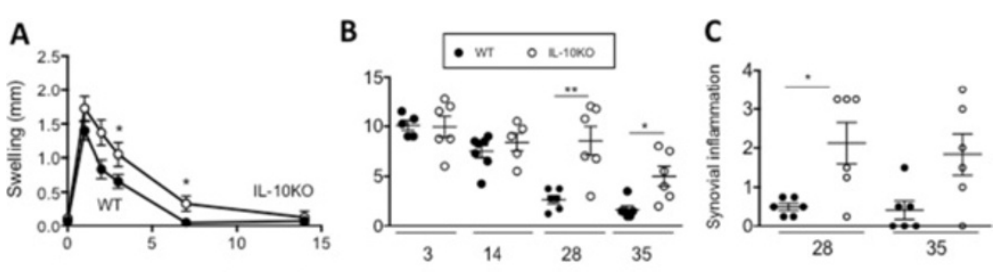

Figure 1 IL-10KO mice have enhanced and prolonged antigen-induced arthritis (AIA). Disease activity in AIA challenged WT (closed circles) and IL-10KO (open circles) mice. (A) Joint swelling, (B) arthritic index scores and (C) synovial inflammation are shown. Swelling data reflects the mean \pm SEM of knee diameters (in $\mathrm{mm} ; n=7-28,{ }^{*} P<0.05$ ). Arthritic index and synovial inflammation values post AIA are presented for individual joints taken at days $3,14,28$ and 35 (data is presented as the mean \pm SEM, $n=5-7$ per time point from a single experiment, ${ }^{*} P<0.05,{ }^{* *} P<0.01$ between WT and IL-10KO groups). IL-10KO, interleukin-10-deficient; WT, wild-type.

was observed around sites of focal bone erosions in sections from AIA-challenged IL-10KO mice (Figure 2B). Radiographic assessments of arthritic joints supported this finding and revealed a more pronounced loss of bone integrity in IL-10KO mice (Figure 2C). To substantiate an involvement of IL-10 in bone turnover, the presence of TRAP-positive osteoclasts was detected (Figure 2D). Quantification of TRAP stain showed an increase in TRAP-positive cells in joint sections from IL-10KO mice (Figure 2D). In accordance, elevated Acp5 (encoding TRAP) gene expression was also observed in synovial mRNA from IL-10KO mice (Figure 2D). This was mirrored by a systemic increase in the surrogate bone erosion marker, DKK-1 (Figure 2D).

\section{Focal bone erosions are associated with localized IL-1 $\beta$ production}

To define the mechanisms governing bone damage in IL$10 \mathrm{KO}$ mice, we next considered the inflammatory environment of the inflamed synovium. Total mRNA was extracted from the synovium of AIA-challenged mice (taken at day 3 post AIA to reflect the initiation of disease) and cytokine gene expression compared in IL-10KO and WT mice. While IL-10KO mice showed no substantive alteration in synovial Il6 or Tnf over 28 days, there was a twofold increase in $I l 1 b$ over that seen in WT mice three days after arthritis induction, which had resolved by day 28 (Figure 3A). Increased synovial IL-1 $\beta$ expression was also observed by immunohistochemistry (Figure 3B). Interleukin-1 $\beta$ protein staining was most prominent in joint sections from IL-10KO mice at day 28 post AIA and coincided with the observed elevation in bone erosion. In this regard, IL- $1 \beta$ staining co-localized to areas of focal bone erosion (Figure 3C). Other IL-1 family members, including IL-18 and IL-33 have also been linked with bone turnover [28,29]. Synovial mRNA from AIA-challenged IL-10KO mice showed no change in Il18 expression, but a 3.5-fold increase in Il33 as compared to WT controls (Figure 3D). These data highlight a specific role of IL-1 family members in the destructive processes of inflammatory arthritis.

\section{IL-10 regulates inflammasome expression within the inflamed synovium}

Activation of the inflammasome is required for the generation of active IL-1 $\beta$. We therefore examined the synovial regulation of inflammasome components by IL-10 during arthritis. At day 3 post AIA induction, synovial Nlrp3, Aim2, Casp1 and Casp12 were all significantly elevated (approximately two- to threefold) in IL-10KO mice when compared to WT controls (Figure 4A). In contrast, Nlrp1, Asc, Rigi, Nod1 and Nod2 remained largely unaltered from WT mice (Figure 4A). To verify a link between IL-10 and synovial inflammasome expression, WT mice were administered (i.a.) with a blocking anti-IL-10R antibody at the onset of AIA (Figure 4B). When compared to isotype or vehicle-control treatments, inhibition of IL-10R signaling promoted a threeto fourfold increase in synovial Nlrp3, Casp1 and Casp12, while Aim2 showed a trend towards significance (Figure 4B). Thus, confirming a link between IL-10 and regulation of the Nlrp3 inflammasome within the inflamed synovium.

\section{Regulation of osteoclastogenesis by IL-10 and the inflammasome}

We next sought to provide a link between arthritic bone erosions and the regulation of the inflammasome. Quantitative PCR revealed a positive correlation between Acp5 and Nlrp3 ( $\mathrm{r}=0.8173)$ and Casp1 $(\mathrm{r}=0.47)$ in samples from IL-10KO mice (Figure 5A). We therefore considered the involvement of IL-10 and the NLRP3 in an in vitro MCSF/ RANKL-driven osteoclast differentiation assay. Consistent with previous studies [13,15], IL-10 blocked osteoclast differentiation by approximately twofold (Figure 5B). Under these conditions, IL-10 inhibited Acp5 and Ctsk (cathepsin-K, which contributes to osteoclastmediated bone resorption) expression (Figure 5B). To assess the role of the inflammasome in this process, osteoclast formation was monitored in the present of cytokine release inhibitory drug-3 (CRID3) or glibenclamide. CRID3 inhibits NLRP3 and AIM2 by blocking ASC oligomers and the activation of caspase 1 [30], while glibenclamide is an 


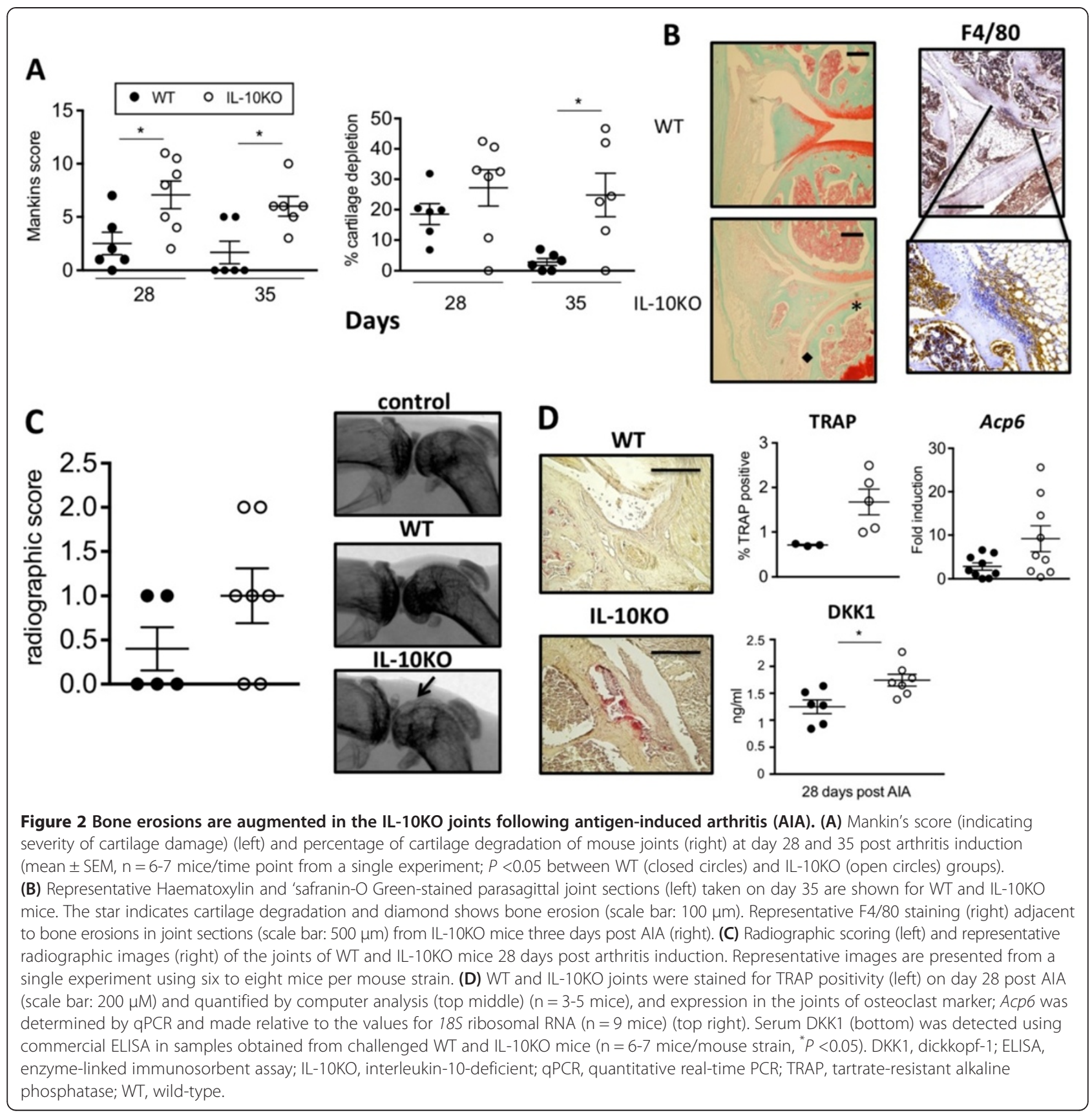

ATP-sensitive potassium channel inhibitor that prevents maturation of caspase 1 and pro-IL-1 $\beta$ through NLRP3 [31]. Using myeloid progenitors from IL-10KO mice, addition of CRID3 or glibenclamide significantly reduced osteoclast numbers and blocked generation of TRAPpositive cells (Figure 5C). These data support a role for NLRP3 in governing bone turnover and erosion.

\section{Discussion}

Innate sensing mechanisms were traditionally linked with the recognition of bacterial, fungal and viral infections.
This viewpoint has, however, changed and many act as sensors of both endogenous and exogenous danger signals [32-34]. Members of the NLR family are intrinsic to caspase-activating complexes termed the inflammasome. These receptors recognize certain infectious pathogens, particles (for example, microcrystals), metabolic anomalies (for example, hyperglycaemia, ATP) and chemicals, and contribute to the pathology of various autoinflammatory diseases [32]. This has led to the novel application of drugs that target IL-1 $\beta$ (for example, anakinra, canakinumab and rilonacept) in conditions such as periodic fever 

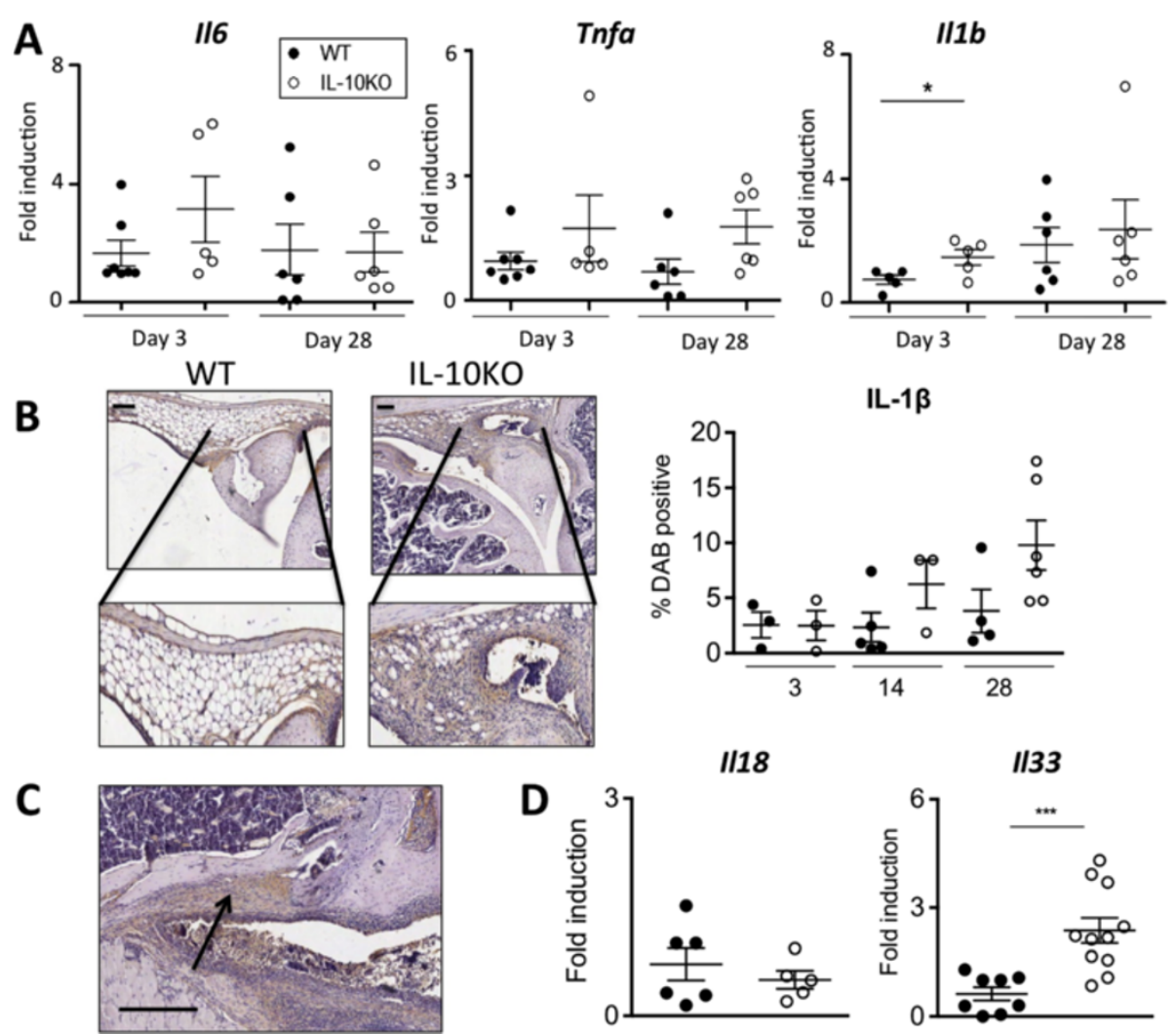

Figure 3 Augmented IL-1 $\beta$ in the IL-10KO mice following antigen-induced arthritis (AIA). (A) Expression of inflammatory cytokines; II6, Tnf and $1 / 16$ were examined in the synovial mRNA of WT (closed circles) and IL-10KO (open circles) mice 3 and 28 days after AIA induction and made relative to the values for 185 ribosomal RNA $\left(n=4-7,{ }^{*} P<0.05\right)$. (B) Representative immunohistochemistry (left) and computer-quantified scoring (right) of IL-1 $\beta$ staining in joint sections (scale bar: $100 \mu \mathrm{m}$ ) for WT and IL-10KO joints. Results show individual joints taken over the course of disease in AIA-challenged mice $(n=3-6)$. (C) Representative immunohistochemical staining for areas of focal IL-1 $\beta$ secretion in IL-10KO bone 28 days post AIA (scale bar: $300 \mu \mathrm{m}$ ). (D) //18 and I/33 were examined in synovial mRNA from WT and IL-10KO mice taken three days after AIA induction. Values were made relative to 185 ribosomal RNA ( $\left.n=5-11,{ }^{* * *} \mathrm{P}<0.001\right)$. IL-10KO, interleukin-10-deficient; WT, wild-type.

syndromes, Still's disease, Schnitzler's syndrome, and gouty arthritis where conventional anti-inflammatory drugs fail to provide long-lasting relief [35]. Here, we show that IL-10 negatively regulates the expression of NLRP3 inflammasome components within the inflamed synovium of experimental arthritis and provide a link to degenerative bone erosion.

Several lines of evidence support an involvement of the inflammasome in inflammatory and degenerative arthritis. While NLRP3 is expressed in RA and OA synovium $[36,37]$ it is difficult to comment on the functional significance of these findings as quantification of transcript levels provide minimal information on inflammasome activity. An involvement of the inflammasome in joint pathology is illustrated by studies of calcium crystals, which accumulate through biomechanical stress or altered mechanisms of calcification. Here, the ectopic deposition of hydroxyapatite crystals in synovial fluids from osteoarthritis patients is associated with disease progression [38]. Hydroxyapatite crystals activate the NLRP3 inflammasome to promote IL-1 $\beta$ and IL-18 release by lipopolysaccharide (LPS)-primed macrophages [24]. Studies in an air pouch model of synovitis also confirmed the activation of NLRP3/ASC/caspase 1 by hydroxyapatite crystals and supported a role in controlling neutrophil infiltration [24]. Analysis of synovial inflammation in AIA-challenged ASC-KO mice showed reduced disease severity [39]. This response was not, however, seen in NLRP3-KO and caspase1-KO mice, where both genotypes showed similar pathology to that observed in WT controls [39]. These results are consistent with our own, where we see similar disease pathology between the WT and IL-10KO mice at these early time points. We now show that at the later stages of inflammatory arthritis, in the absence of IL-10, joint inflammation results in a temporal increase in synovial IL-1 $\beta$, which corresponds with enhanced pathology and colocalization to areas rich in F4/80-postive cells that cluster at sites of focal bone erosion. Such changes in IL-1 $\beta$ may reflect the capacity of IL-10 to inhibit TLR control of Il1 $\beta$ (pro-IL-1 $\beta$ ) and the expression of NLRP3 inflammasome components. At this stage we are, however, unable to ascertain what inflammatory events (for example, 


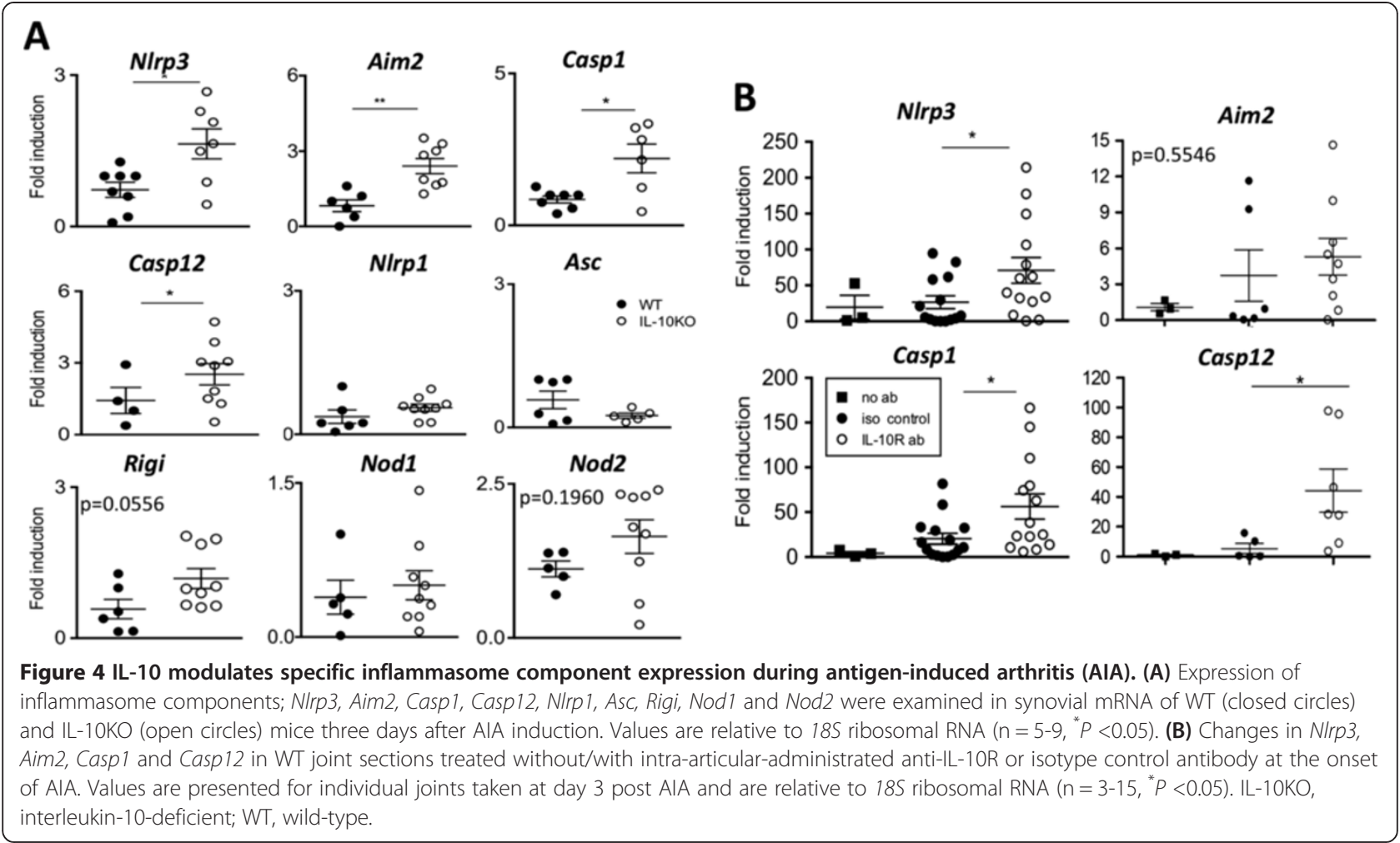

TLR or cytokine-driven outcomes) regulate the induction of these inflammasome genes.

Our results implicate the involvement of the inflammasome in osteoclastogenesis, with IL-10 deficiency causing an increase in both synovial IL-1 $\beta$ and IL-33 expression, but not IL-18. Interleukin- $1 \beta$ is considered pro-osteoclastogenic and drives an imbalance in chondrocyte responses through regulation of prostanoids, nitric oxide and other free radicals, and the induction of degradative enzymes that impact collagen and proteoglycan turnover $[40,41]$. In contrast, IL-33 and IL-18 are considered anti-osteoclastogenic and protect against tumour necrosis factor alpha (TNF $\alpha$ )-mediated bone loss $[42,43]$. Thus, IL-33 often opposes the activities of IL-1 $\beta$, which raises a question about the regulation of these cytokines by the NLRP3/caspase 1 system. While pro-IL- $1 \beta$ and pro-IL-18 are processed by caspase 1 into mature active forms, full-length IL-33 is already biologically active and is released as a consequence of cell damage [44]. Further processing by caspase 1 causes inactivation of IL-33 [44,45]. IL-33 is also modified by the activity of elastase and cathepsin G, which are secreted by infiltrating neutrophils and enhance IL-33 bioactivity [46]. Here, IL-33 acts as an endogenous danger signal (alarmin), which alerts innate immune cells to sites of infection or injury. Thus, the elevated expression of Il33 in IL-10KO mice may simply reflect the overall increase in synovial inflammation. Moreover, the blockade of osteoclastogenesis by CRID3 and glibenclamide suggests a role for caspase 1 in bone turnover. Targeting the inflammasome may therefore benefit joint pathologies allied with IL- $1 \beta$ production, such as OA, MuckleWells syndrome (an autoinflammatory disorder linked with mutations in NLRP3) and gout $[20,35,47,48]$. Such an approach may provide an added benefit over traditional biologic interventions including anakinra, which in clinical trials of OA offered no improvement in symptoms [49]. To provide preclinical evidence to support this notion, attempts were made to treat AIAchallenged IL-10KO mice (i.a.) with either CRID3 or glibenclamide. However, administration (i.a.) of vehicle alone as a control promoted a robust inflammatory response, which prevented the direct investigation of this in vivo.

Our results indicate that IL-10 has the capacity to inhibit expression of NLRP3 and certain components of the inflammasome. Here, neutralization of IL-10 receptor signaling in WT mice enhanced synovial Nlrp3 and Casp1 expression and addition of endogenous IL-10 inhibited the formation of TRAP+ osteoclasts in vitro. Significantly, human trials with recombinant IL-10 showed no improvement in disease activity [50], while IL-10 responses in synovial macrophages from RA patients appear dysregulated [51]. Such alterations in IL-10 responsiveness results in a loss of its antiinflammatory properties and a concomitant acquisition of interferon-gamma (IFNY)-like activities [51]. 


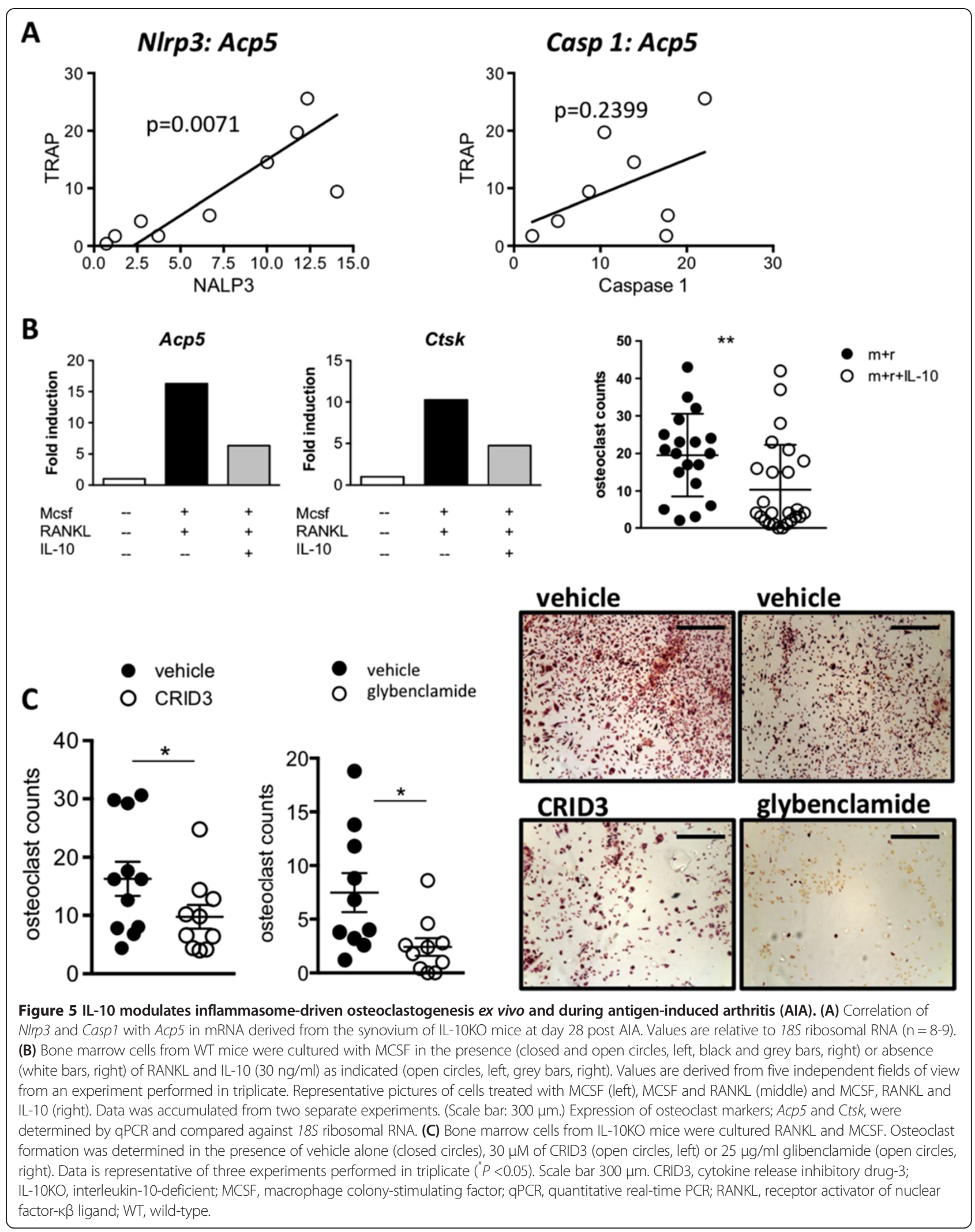




\section{Conclusions}

Our studies implicate a link between IL-10 and expression of the NLRP3 inflammasome within the inflamed synovium, and advocate a role for IL-10 and inflammasome activation in governing osteoclastogenesis and bone erosion. With advances in synovial histopathology taking centre stage in clinical practice [2], monitoring IL-10 or inflammasome activities within the inflamed joints of patients with early RA may prove valuable as a predictor of disease activity or bone erosions, and may help tailor the design of novel treatments for defined patient groups.

\section{Additional file}

\section{Additional file 1: Oligonucleotide primer sequences for real-time}

PCR. Oligonucleotide primer sequences for each of the inflammatory mediators measured in the study. All sequences are listed in the $5^{\prime}-3^{\prime}$ direction.

\section{Abbreviations}

AIA: antigen-induced arthritis; (M)CFA: (modified) Freud's complete adjuvant; CRID3: cytokine release inhibitory drug-3; DKK-1: dickkopf-1;

ELISA: enzyme-linked immunosorbent assay; i.a.: intra-articular; IL-10 (KO): interleukin-10(-deficient); i.p.: intraperitoneally; mBSA: methylated bovine serum albumin; MCSF: macrophage colony-stimulating factor; NLR: nucleotide-binding domain and leucine-rich repeat-containing receptors; OA: osteoarthritis; PBS: phosphate-buffered saline; qPCR: quantitative real-time PCR; RA: rheumatoid arthritis; RANKL: receptor activator of nuclear factor-k $\beta$ ligand; RLR: Rig-Ilike receptors; $S$. c.: subcutaneous; TLR: Toll-like receptors; TRAP: tartrate-resistant alkaline phosphatase; WT: wild-type.

\section{Competing interests}

The authors declare that they have no competing interests.

\section{Authors' contributions}

CJG, GWJ, MAN and ASW performed all animal experiments, tissue processing, histology scoring, and contributed to the drafting of the manuscript. CJG and ZN performed all real-time PCR and immunoassays. AKH, ANM and MAN participated in the radiographic studies and design of scoring criteria. CJG, AKH, FLC, ACB, ASW and MR performed all osteoclast studies including data analysis and assay development. AKH participated in the statistical evaluation of data. RC, LAO, MAC and IRH provided necessary research reagents and AABR synthesized the CRID3. RC, LAO and MAC provide necessary technical expertise relating to the inhibition of the inflammasome and interpretation of results. PRT, IRH, ASW and SAJ designed experiments and evaluated all results. CJG, GWJ and SAJ wrote the manuscript. All authors read and approved the manuscript.

\section{Authors' information}

IRH is recipient of a Wellcome Trust Senior Fellowship in Basic Biomedical Science, and PRT holds an MRC Senior Fellowship (G0601617). Funding for this research was provided by Arthritis Research UK fellowships 19234 and 20305 (to GWJ), PhD Studentship 18598 (to ASW) and grants 19796, 19381, 18286 (to SAJ).

\section{Acknowledgements}

Research funding was provided by Arthritis Research UK fellowships 19234 and 20305 (to GWJ) and grants 19796, 19381, 18286 (to SAJ).

\section{Author details}

${ }^{1}$ Cardiff Institute of Infection and Immunity, The School of Medicine, Cardiff University, Heath Park Campus, Cardiff CF14 4XN, Wales, UK. ${ }^{2}$ Trinity Biomedical Sciences Institute, School of Biochemistry and Immunology, Trinity College Dublin, 152-160 Pearse Street, Dublin 2, Ireland. ${ }^{3}$ Institute for Molecular Bioscience, The University of Queensland, St Lucia Campus, Brisbane 4072, QLD, Australia.
Received: 29 November 2013 Accepted: 1 August 2014

Published online: 30 August 2014

\section{References}

1. Choy E: Understanding the dynamics: pathways involved in the pathogenesis of rheumatoid arthritis. Rheumatology 2012, 51:v3-v11.

2. Choy EH, Kavanaugh AF, Jones SA: The problem of choice: current biologic agents and future prospects in RA. Nat Rev Rheumatol 2013, 9:154-163.

3. Kumar H, Kawai T, Akira S: Pathogen recognition by the innate immune system. Int Rev Immunol 2011, 30:16-34.

4. Sancho D, Reise Sousa C: Signaling by myeloid C-type lectin receptors in immunity and homeostasis. Annu Rev Immunol 2012, 30:491-529.

5. Fiorentino DF, Zlotnik A, Mosmann TR, Howard M, O'Garra A: IL-10 inhibits cytokine production by activated macrophages. J Immunol 1991, 147:3815-3822.

6. Moore KW, O'Garra A, De Waal Malefyt R, Vieira P, Mosmann TR: Interleukin-10. Annu Rev Immunol 1993, 11:165-190.

7. Dagvadorj J, Naiki Y, Tumurkhuu G, Hassan F, Islam S, Koide N, Mori I, Yoshida T, Yokochi T: Interleukin-10 inhibits tumor necrosis factor-alpha production in lipopolysaccharide-stimulated RAW 264.7 cells through reduced MyD88 expression. Innate Immun 2008, 14:109-115.

8. Chang J, Kunkel SL, Chang CH: Negative regulation of MyD88-dependent signaling by IL-10 in dendritic cells. Proc Natl Acad Sci U S A 2009, 106:18327-18332.

9. Finnegan A, Kaplan CD, Cao Y, Eibel H, Glant TT, Zhang J: Collagen-induced arthritis is exacerbated in IL-10-deficient mice. Arthritis Res Ther 2003, 5:R18-R24.

10. Walmsley M, Katsikis PD, Abney E, Parry S, Williams RO, Maini RN, Feldmann $\mathrm{M}$ : Interleukin-10 inhibition of the progression of established collageninduced arthritis. Arthritis Rheum 1996, 39:495-503.

11. Couper KN, Blount DG, Riley EM: IL-10: the master regulator of immunity to infection. J Immunol 2008, 180:5771-5777.

12. Mocellin S, Marincola F, Rossi CR, Nitti D, Lise M: The multifaceted relationship between IL-10 and adaptive immunity: putting together the pieces of a puzzle. Cytokine Growth Factor Rev 2004, 15:61-76.

13. Evans KE, Fox SW: Interleukin-10 inhibits osteoclastogenesis by reducing NFATc1 expression and preventing its translocation to the nucleus. BMC Cell Biol 2007, 8:4.

14. Lettesjo H, Nordstrom E, Strom H, Nilsson B, Glinghammar B, Dahlstedt L, Moller E: Synovial fluid cytokines in patients with rheumatoid arthritis or other arthritic lesions. Scand J Immunol 1998, 48:286-292.

15. Owens JM, Gallagher AC, Chambers TJ: IL-10 modulates formation of osteoclasts in murine hemopoietic cultures. J Immunol 1996, 157:936-940.

16. Schroder K, Tschopp J: The inflammasomes. Cell 2010, 140:821-832.

17. Gracie JA, Forsey RJ, Chan WL, Gilmour A, Leung BP, Greer MR, Kennedy K, Carter R, Wei XQ, Xu D, Field M, Foulis A, Liew FY, Mclnnes IB: A proinflammatory role for IL-18 in rheumatoid arthritis. J Clin Invest 1999, 104:1393-1401.

18. Kay J, Calabrese L: The role of interleukin- 1 in the pathogenesis of rheumatoid arthritis. Rheumatology 2004, 43:iii2-iii9.

19. Halverson PB, McCarty DJ: Identification of hydroxyapatite crystals in synovial fluid. Arthritis Rheum 1979, 22:389-395.

20. Martinon F, Petrilli V, Mayor A, Tardivel A, Tschopp J: Gout-associated uric acid crystals activate the NALP3 inflammasome. Nature 2006, 440:237-241.

21. Richette P, Bardin T, Doherty M: An update on the epidemiology of calcium pyrophosphate dihydrate crystal deposition disease. Rheumatology 2009, 48:711-715.

22. Carroll GJ, Stuart RA, Armstrong JA, Breidahl PD, Laing BA: Hydroxyapatite crystals are a frequent finding in osteoarthritic synovial fluid, but are not related to increased concentrations of keratan sulfate or interleukin 1 beta. J Rheumatol 1991, 18:861-866

23. Whelan LC, Morgan MP, McCarthy GM: Basic calcium phosphate crystals as a unique therapeutic target in osteoarthritis. Front Biosci 2005, 10:530-541.

24. Jin C, Frayssinet P, Pelker R, Cwirka D, Hu B, Vignery A, Eisenbarth SC, Flavell RA: NLRP3 inflammasome plays a critical role in the pathogenesis of hydroxyapatite-associated arthropathy. Proc Natl Acad Sci U S A 2011, 108:14867-14872. 
25. Pazar B, Ea HK, Narayan S, Kolly L, Bagnoud N, Chobaz V, Roger T, Liote F, So A, Busso N: Basic calcium phosphate crystals induce monocyte/ macrophage IL-1beta secretion through the NLRP3 inflammasome in vitro. J Immunol 2011, 186:2495-2502.

26. Mathews RJ, Robinson Jl, Battellino M, Wong C, Taylor JC, Eyre S, Churchman SM, Wilson AG, Isaacs JD, Hyrich K, Barton A, Plant D, Savic S, Cook GP, Sarzi-Puttini P, Emery P, Barrett JH, Morgan AW, McDermott MF: Evidence of NLRP3-inflammasome activation in rheumatoid arthritis (RA); genetic variants within the NLRP3-inflammasome complex in relation to susceptibility to RA and response to anti-TNF treatment. Ann Rheum Dis 2013, 73:1202-1210.

27. Evans L, Williams AS, Hayes AJ, Jones SA, Nowell M: Suppression of leukocyte infiltration and cartilage degradation by selective inhibition of pre-B cell colony-enhancing factor/visfatin/nicotinamide phosphoribosyltransferase: Apo866-mediated therapy in human fibroblasts and murine collagen-induced arthritis. Arthritis Rheum 2011, 63:1866-1877.

28. Dai SM, Nishioka K, Yudoh K: Interleukin (IL) 18 stimulates osteoclast formation through synovial $T$ cells in rheumatoid arthritis: comparison with IL1 beta and tumour necrosis factor alpha. Ann Rheum Dis 2004, 63:1379-1386

29. Mun SH, Ko NY, Kim HS, Kim JW, Kim Do K, Kim AR, Lee SH, Kim YG, Lee CK, Kim BK: Interleukin-33 stimulates formation of functional osteoclasts from human CD14(+) monocytes. Cell Mol Life Sci 2010, 67:3883-3892.

30. Coll RC, O'Neill LA: The cytokine release inhibitory drug CRID3 targets ASC oligomerisation in the NLRP3 and AIM2 inflammasomes. PLOS One 2011, 6:e29539.

31. Lamkanfi M, Mueller JL, Vitari AC, Misaghi S, Fedorova A, Deshayes K, Lee WP, Hoffman HM, Dixit VM: Glyburide inhibits the cryopyrin/Nalp3 inflammasome. J Cell Biol 2009, 187:61-70.

32. Heine H: TLRs, NLRs and RLRs: innate sensors and their impact on allergic diseases-a current view. Immunol Lett 2011, 139:14-24.

33. Mariathasan S, Weiss DS, Newton K, McBride J, O'Rourke K, Roose-Girma M, Lee WP, Weinrauch Y, Monack DM, Dixit VM: Cryopyrin activates the inflammasome in response to toxins and ATP. Nature 2006, 440:228-232.

34. Yamasaki K, Muto J, Taylor KR, Cogen AL, Audish D, Bertin J, Grant EP, Coyle AJ, Misaghi A, Hoffman HM, Gallo RL: NLRP3/cryopyrin is necessary for interleukin-1 beta (IL-1beta) release in response to hyaluronan, an endogenous trigger of inflammation in response to injury. J Biol Chem 2009, 284:12762-12771.

35. Dinarello CA, Simon A, van der Meer JW: Treating inflammation by blocking interleukin-1 in a broad spectrum of diseases. Nat Rev Drug Discov 2012, 11:633-652.

36. Kolly L, Busso N, Palmer G, Talabot-Ayer D, Chobaz V, So A: Expression and function of the NALP3 inflammasome in rheumatoid synovium. Immunology 2010, 129:178-185

37. Rosengren S, Hoffman HM, Bugbee W, Boyle DL: Expression and regulation of cryopyrin and related proteins in rheumatoid arthritis synovium. Ann Rheum Dis 2005, 64:708-714.

38. McCarthy GM, Cheung HS: Point: Hydroxyapatite crystal deposition is intimately involved in the pathogenesis and progression of human osteoarthritis. Curr Rheumatol Rep 2009, 11:141-147.

39. Kolly L, Karababa M, Joosten LA, Narayan S, Salvi R, Petrilli V, Tschopp J, van den Berg WB, So AK, Busso N: Inflammatory role of ASC in antigeninduced arthritis is independent of caspase-1, NALP-3, and IPAF. $\mathrm{J}$ Immunol 2009, 183:4003-4012.

40. Pelletier JP, Martel-Pelletier J, Abramson SB: Osteoarthritis, an inflammatory disease: potential implication for the selection of new therapeutic targets. Arthritis Rheum 2001, 44:1237-1247.

41. Chevalier X: Upregulation of enzymatic activity by interleukin-1 in osteoarthritis. Biomed Pharmacother 1997, 51:58-62.

42. Udagawa N, Horwood NJ, Elliott J, Mackay A, Owens J, Okamura H, Kurimoto M, Chambers TJ, Martin TJ, Gillespie MT: Interleukin-18 (interferon-gamma-inducing factor) is produced by osteoblasts and acts via granulocyte/macrophage colony-stimulating factor and not via interferon-gamma to inhibit osteoclast formation. J Exp Med 1997, 185:1005-1012.

43. Zaiss MM, Kurowska-Stolarska M, Bohm C, Gary R, Scholtysek C, Stolarski B, Reilly J, Kerr S, Millar NL, Kamradt T, McInnes IB, Fallon PG, David JP, Liew FY, Schett G: IL-33 shifts the balance from osteoclast to alternatively activated macrophage differentiation and protects from TNF-alphamediated bone loss. J Immunol 2011, 186:6097-6105.
44. Lefrancais E, Cayrol C: Mechanisms of IL-33 processing and secretion: differences and similarities between IL-1 family members. Eur Cytokine Netw 2012, 23:120-127.

45. Cayrol C, Girard JP: The IL-1-like cytokine IL-33 is inactivated after maturation by caspase-1. Proc Natl Acad Sci U S A 2009, 106:9021-9026.

46. Lefrancais E, Roga S, Gautier V, Gonzalez-de-Peredo A, Monsarrat B, Girard JP, Cayrol C: IL-33 is processed into mature bioactive forms by neutrophil elastase and cathepsin G. Proc Natl Acad Sci U S A 2012, 109:1673-1678.

47. Agostini L, Martinon F, Burns K, McDermott MF, Hawkins PN, Tschopp J: NALP3 forms an IL-1 beta-processing inflammasome with increased activity in Muckle-Wells autoinflammatory disorder. Immunity 2004, 20:319-325.

48. Daheshia M, Yao JQ: The interleukin 1beta pathway in the pathogenesis of osteoarthritis. J Rheumatol 2008, 35:2306-2312.

49. Chevalier X, Goupille P, Beaulieu AD, Burch FX, Bensen WG, Conrozier T, Loeuille D, Kivitz AJ, Silver D, Appleton BE: Intraarticular injection of anakinra in osteoarthritis of the knee: a multicenter, randomized, double-blind, placebo-controlled study. Arthritis Rheum 2009, 61:344-352.

50. van Roon J, Wijngaarden S, Lafeber FP, Damen C, van de Winkel J, Bijlsma JW: Interleukin 10 treatment of patients with rheumatoid arthritis enhances Fc gamma receptor expression on monocytes and responsiveness to immune complex stimulation. J Rheumatol 2003, 30:648-651.

51. Antoniv TT, Ivashkiv LB: Dysregulation of interleukin-10-dependent gene expression in rheumatoid arthritis synovial macrophages. Arthritis Rheum 2006, 54:2711-2721.

doi:10.1186/s13075-014-0419-y

Cite this article as: Greenhill et al.: Interleukin-10 regulates the inflammasome-driven augmentation of inflammatory arthritis and joint destruction. Arthritis Research \& Therapy 2014 16:419.

\section{Submit your next manuscript to BioMed Central and take full advantage of:}

- Convenient online submission

- Thorough peer review

- No space constraints or color figure charges

- Immediate publication on acceptance

- Inclusion in PubMed, CAS, Scopus and Google Scholar

- Research which is freely available for redistribution

Submit your manuscript at www.biomedcentral.com/submit
C BioMed Central 